\title{
On the Efficiency of NHS Ester Cross-Linkers for Stabilizing Integral Membrane Protein Complexes
}

\author{
Fan Chen, ${ }^{1}$ Sabina Gerber, ${ }^{2}$ Volodymyr M. Korkhov, ${ }^{2}$ Samantha Mireku, ${ }^{2}$ Monika Bucher, ${ }^{2}$ \\ Kaspar P. Locher, ${ }^{2}$ Renato Zenobi ${ }^{1}$
}

${ }^{1}$ Department of Chemistry and Applied Biosciences, ETH Zürich, 8093 Zürich, Switzerland

${ }^{2}$ Institute of Molecular Biology and Biophysics, ETH Zürich, 8093 Zürich, Switzerland

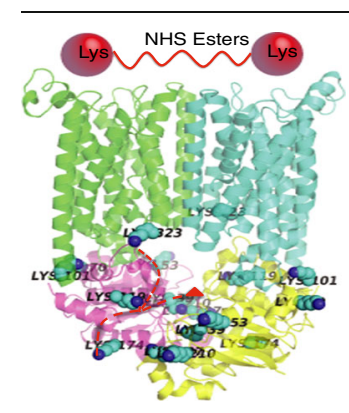

\begin{abstract}
We have previously presented a straightforward approach based on highmass matrix-assisted laser desorption/ionization (MALDI) mass spectrometry (MS) to study membrane proteins. In addition, the stoichiometry of integral membrane protein complexes could be determined by MALDI-MS, following chemical crosslinking via glutaraldehyde. However, glutaraldehyde polymerizes in solution and reacts nonspecifically with various functional groups of proteins, limiting its usefulness for structural studies of protein complexes. Here, we investigated the capability of $N$-hydroxysuccinimide (NHS) esters, which react much more specifically, to crosslink membrane protein complexes such as PgIK and $B t u C^{2} D^{2}$. We present clear evidence that NHS esters are capable of stabilizing membrane protein complexes in
\end{abstract} situ, in the presence of detergents such as DDM, C12E8, and LDAO. The stabilization efficiency strongly depends on the membrane protein structure (i.e, the number of primary amine groups and the distances between primary amines). A minimum number of primary amine groups is required, and the distances between primary amines govern whether a cross-linker with a specific spacer arm length is able to bridge two amine groups. Key words: Membrane protein complexes, MALDI, Chemical cross-linking, NHS-esters

Received: 19 August 2014/Revised: 20 October 2014/Accepted: 21 October 2014/Published Online: 18 November 2014

\section{Introduction}

$\mathrm{M}$ ass spectrometry (MS), using electrospray ionization (ESI) or matrix-assisted laser desorption/ionization (MALDI), is a powerful method for studying macromolecular complexes. Membrane proteins, however, are difficult to study by MS because detergents are required for solubilizing them, which often compromises efficient ionization. Marcoux and Robinsin have written a good review on recent progress in studying membrane proteins and their complexes by native ESI-MS [1]. The first mass spectrum of a membrane protein complex in detergent micelles recored by native ESI-MS was the heteromeric vitamin $\mathrm{B}_{12}$ importer $\mathrm{BtuC}_{2} \mathrm{D}_{2}$ [2]. Laserinduced liquid bead ion desorption (LILBID) MS, a highly specialized technique, was also applied to study membrane protein complexes, specifically the oligomeric state of ExbB

Electronic supplementary material The online version of this article (doi:10.1007/s13361-014-1035-4) contains supplementary material, which is available to authorized users.

Correspondence to: Renato Zenobi; e-mail: zenobi@org.chem.ethz.ch and ExbB-ExbD [3]. Alternatively, MALDI-MS with highmass detection capabilities is a straighforward method to study integral membrane proteins [4]. It allows rapid determination of their molecular weights, pinpointing glycosylation sites, and elucidation of the subunit stoichiometry of membrane protein complexes, without the need for extensive sample purification and optimization of the sample preparation [4].

Noncovalent interactions are easily disrupted in MALDI, either during sample preparation or ion formation. Chemical cross-linkers such as glutaraldehyde are thus often used, to stabilize noncovalent interactions before analyzing complexes by MALDI-MS [5, 6]. Although glutaraldehyde is known to react with membrane protein complexes [4], the structure of glutaraldehyde in aqueous solution is not well defined because it polymerizes. Moreover, glutaraldehyde reacts unspecifically with a number of functional groups of proteins [7], which severely limits its application in structure determination. For instance, different polymeric forms of glutaraldehyde compromise mapping the distances between different amino acid side chains, which is at the core of threedimensional structural analysis based on chemical crosslinking combined with MS. 
$N$-hydroxysuccinimide (NHS) esters, which react specifically with Lys residues, are among the most widely applied chemical cross-linkers. They are convenient for analyzing the three-dimensional structure of proteins because of the high prevalence of lysine residues in proteins (about 6\%). Under carefully controlled reaction conditions, side reactions of NHS esters with amino acids other than Lys can be largely avoided [8]. Cross-linking protocols, mass spectrometric analysis of cross-linked samples, and also data analysis are well established, as described in some recent reviews [9-12].

Recently, NHS esters have been applied in structure characterization of membrane proteins [13-16]. It has been reported that a NHS ester-based cross-linker, which was used in the development of the so-called protein interaction reporter (PIR) technology, was able to stabilize protein complexes in living cells, including outer membrane protein A (OmpA) $[13,14]$. Another NHS-based cross-linker, BS3, has also been applied to study chloroplast F-ATPases. The results suggested relations among phosphorylation, dynamic interactions, and regulation of a transmembrane molecular motor $[15,16]$. To futher subject cross-linked proteins to tandem mass spectrometry (top-down approach) or to in-solution digestion (bottom-up approach) for structure determination, it is thus critical to estabilish under which conditions NHS esters react effectively with membrane proteins (or their complexes), in particular in the presence of detergent micelles.

To answer this question, we used a series of NHS esters and two membrane protein complexes, specifically, the ATP binding cassette $(\mathrm{ABC})$ transporters $[17,18] \mathrm{PglK}$ and $\mathrm{BtuC}_{2} \mathrm{D}_{2}$. In the following, we look at the reactivity of NHS-esters with integral membrane protein complexes from two main perspectives, the chemical properties of the cross-linker and the structural properties of the membrane proteins. All four NHS esterbased cross-linkers studied here, including bis(sulfosuccinimidyl) suberate $\left(\mathrm{BS}^{3}\right)$, disuccinimidyl suberate (DSS), bis(succinimidyl) penta(ethylene glycol) $\left(\mathrm{BS}(\mathrm{PEG})_{5}\right)$, and bis(succinimidyl) nonaethyleneglycol (BS(PEG) 9 ) (Supplementary Table 1) were found to be capable of stabilizing membrane protein complexes in situ. The stabilization efficiency strongly depended on the protein structure, including the primary and tertiary structure. We succeeded in cross-linking PglK in the presence of detergents including DDM and C12E8, which are frequently used above the critical micelle concentration for solubilizing membrane proteins.

\section{Materials and Methods}

\section{Materials}

PglK and $\mathrm{BtuC}_{2} \mathrm{D}_{2}$ were purified as described previously [4, 19]. After buffer exchange, $\mathrm{PglK}$ was in a buffer of $10 \mathrm{mM}$ Bicine- $\mathrm{NaOH}$ at $\mathrm{pH} 8.2$ containing $500 \mathrm{mM} \mathrm{NaCl}, 0.5 \mathrm{mM}$ EDTA-NaOH, $10 \%(\mathrm{w} / \mathrm{v})$ glycerol, and $0.016 \%(\mathrm{w} / \mathrm{v})$ n-dodecyl- $\beta$-D-maltopyranoside (DDM, Anatrace). Dodecyl octaethylene glycol ether (C12E8) was also used as a detergent to solublilize PglK. The $\mathrm{BtuC}_{2} \mathrm{D}_{2}$ protein was in a final buffer with the following composition: $50 \mathrm{mM}$ Na-phosphate, $\mathrm{pH} 7$, $500 \mathrm{mM} \mathrm{NaCl}, 0.5 \%$ EDTA, 0.1\% LDAO. Sinapinic acid (SA) was purchased from Sigma-Aldrich Chemie GmbH (Buchs, Switzerland). All chemical cross-linkers were purchased from Pierce Protein Research Products (Thermo Fisher Scientific, Rockford, IL, USA). Trifluoroacetic acid (TFA) was obtained from Acros Organics (Thermo Fisher Scientific, Geel, Belgium). All commercial reagents and solvents were obtained in the highest purity available and used without further purification.

\section{Chemical Cross-Linking Protocol}

Cross-linker solutions were prepared at a concentration 1000 times higher than that of the protein complexes. $\mathrm{BS}^{3}$ was dissolved in water, whereas the other cross-linkers used here were dissolved in acetonitrile. To chemically cross-link the proteins, the cross-linker solutions were mixed with the protein solution in a 1:10 volume ratio at room temperature, for $2 \mathrm{~h}$. The mixture was further diluted with the original protein buffer solution or water prior to mass spectrometric analysis.

\section{Mass Spectrometry}

A commercial MALDI-TOF/TOF mass spectrometer (model 4800 plus; AB SCIEX, Darmstadt, Germany) equipped with a high-mass detector (HM2; CovalX AG, Zurich, Switzerland) was used. All measurements were performed in linear positive ion mode, with standard settings. Ionization was achieved with a Nd:YAG laser $(355 \mathrm{~nm})$ with the pulse energy set just above the threshold for ion formation. Each mass spectrum was the average of 1000 laser shots acquired at random sample positions. Sinapinic acid $(20 \mathrm{mg} / \mathrm{mL}$ in water/acetonitrile/TFA, $49.95 / 49.95 / 0.1, \mathrm{v} / \mathrm{v} / \mathrm{v})$ was used as the matrix. The samples were directly mixed with the matrix solution in a $1 / 2(\mathrm{v} / \mathrm{v})$ ratio. One $\mu \mathrm{L}$ of the mixture was spotted on to a stainless steel plate and allowed to dry under ambient conditions. All mass spectra were baseline-corrected and smoothed using a Savitzky-Golay algorithm available within Igor Pro (ver. 6.2; WaveMetrics, Portland, OR, USA). Distance calculations between specific amino acid residues, based on the protein structure from the Protein Data Bank, were carried out using UCSF Chimera (ver. 1.6.2; University of California, San Francisco, CA, USA).

\section{Results and Discussion}

Our primary aim was to investigate whether NHS esters, which are widely used to map the three-dimensional structure of soluble proteins, are able to cross-link membrane proteins (and their complexes) efficiently in detergent micelles. The structure of PglK [18], a Campylobacter jejuni-encoded ABC transporter, is still not well understood; it is supposed to be a structural homolog of Sav1866, a well-defined homodimeric multidrug $\mathrm{ABC}$ transporter consisting of 12 transmembrane helices [19]. In an earlier study from our laboratory, the oligomeric state of PglK was characterized experimentally for the 
first time via high-mass MALDI-MS, following cross-linking with glutaraldehyde [4]. The monomeric species of PglK at $\mathrm{m} / \mathrm{z}$ 67,500 (theoretical mass $67,327 \mathrm{Da}$, mass error $=0.2 \%$ ) was detected as the dominant peak in the absence of any chemical cross-linking (Figure 1a). After cross-linking with $\mathrm{BS}^{3}$ in the presence of detergent, the signal at $\mathrm{m} / \mathrm{z} 144,500$ corresponding to the dimer becomes dominant (Figure 1b). The molecular weight observed here is larger than the theoretical mass of the PglK dimer (134,654 Da), which is due to $\mathrm{BS}^{3}$ "decoration" as also observed previously [4]. The mass was previously found to increase to $150,900 \mathrm{Da}$ following cross-linking with glutaraldehyde [4], although glutaraldehyde has a lower molecular weight than $\mathrm{BS}^{3}$. The nonspecific reactivity and the involvement of glutaraldehyde oligomers lead to this large molecular weight increase. The observation of a homodimer instead of the monomer after cross-linking suggests that PglK forms a noncovalent dimer in DDM detergent solution and that these noncovalent interactions can be preserved in MALDI after cross-linking with $\mathrm{BS}^{3}$. Dominant dimer species are also observed after crosslinking with $\mathrm{BS}(\mathrm{PEG})_{5}$ (Figure 1c) and $\mathrm{BS}(\mathrm{PEG})_{9}$ (Figure 1d). To further study chemical cross-linking in situ, we carried out cross-linking of PglK in another detergent micelle solution, C12E8. DDM (dodecyl- $\beta$-D-maltoside) and C12E8 (octaethyleneglycol monododecyl ether) are both nonionic detergents that are often used to solubilize and stabilize membrane proteins. The observation of the monomeric species of PglK and a low intensity of PglK dimer in C12E8 suggests that high-mass MALDI-MS can tolerate detergent present in the sample (Figure 2a). Again, we observed the dimer of PglK as the dominant species after chemical crosslinking with $\mathrm{BS}^{3}$ (Figure 2b), DSS (Figure 2c), BS(PEG) (Figure 2d), and BS(PEG) $_{9}$ (Figure 2e). Successful cross-linking of PglK in different detergent micelles, either DDM or C12E8, suggests that NHS esters are capable of stabilizing membrane protein complexes in situ, in the presence of various detergent micelles.

We also studied in-situ cross-linking of $\mathrm{BtuC}_{2} \mathrm{D}_{2}$, a vitamin $\mathrm{B}_{12}$ importer from Escherichia coli [20], with NHS esters and in the presence LDAO. In the absence of any cross-linker, we observed the BtuD subunit at $m / z 26,800$ as the dominant species, and the BtuC subunit at $m / z$ 37,200 with a much lower intensity (Figure 3a). Surprisingly, cross-linking of $\mathrm{BtuC}_{2} \mathrm{D}_{2}$ with $\mathrm{BS}^{3}$ or DSS did not yield any peak corresponding to the complex of $\mathrm{BtuC}_{2} \mathrm{D}_{2}$ (Figure $3 \mathrm{~b}$ and c). However, the molecular weight of the BtuD subunit increased with both crosslinking reagents, suggesting that the cross-linker did react with the amine groups in the BtuD subunit. Only after adding

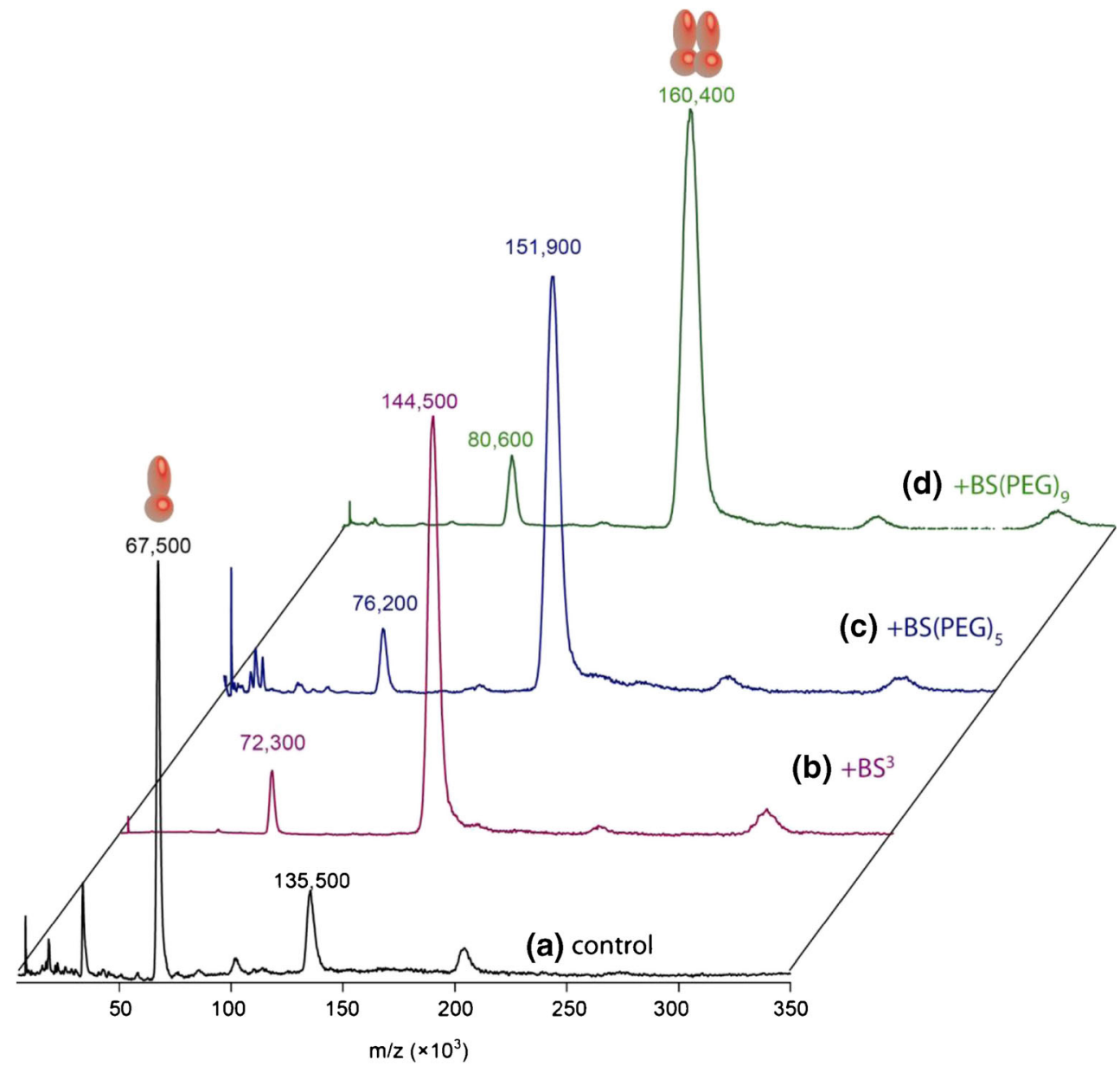

Figure 1. High-mass MALDI mass spectra of PgIK in DDM (a) without any cross-linker, and after applying BS ${ }^{3}(\mathbf{b}), B S(P E G)_{5}(\mathbf{c})$, and $\mathrm{BS}(\mathrm{PEG})_{9}(\mathbf{d})$, respectively 


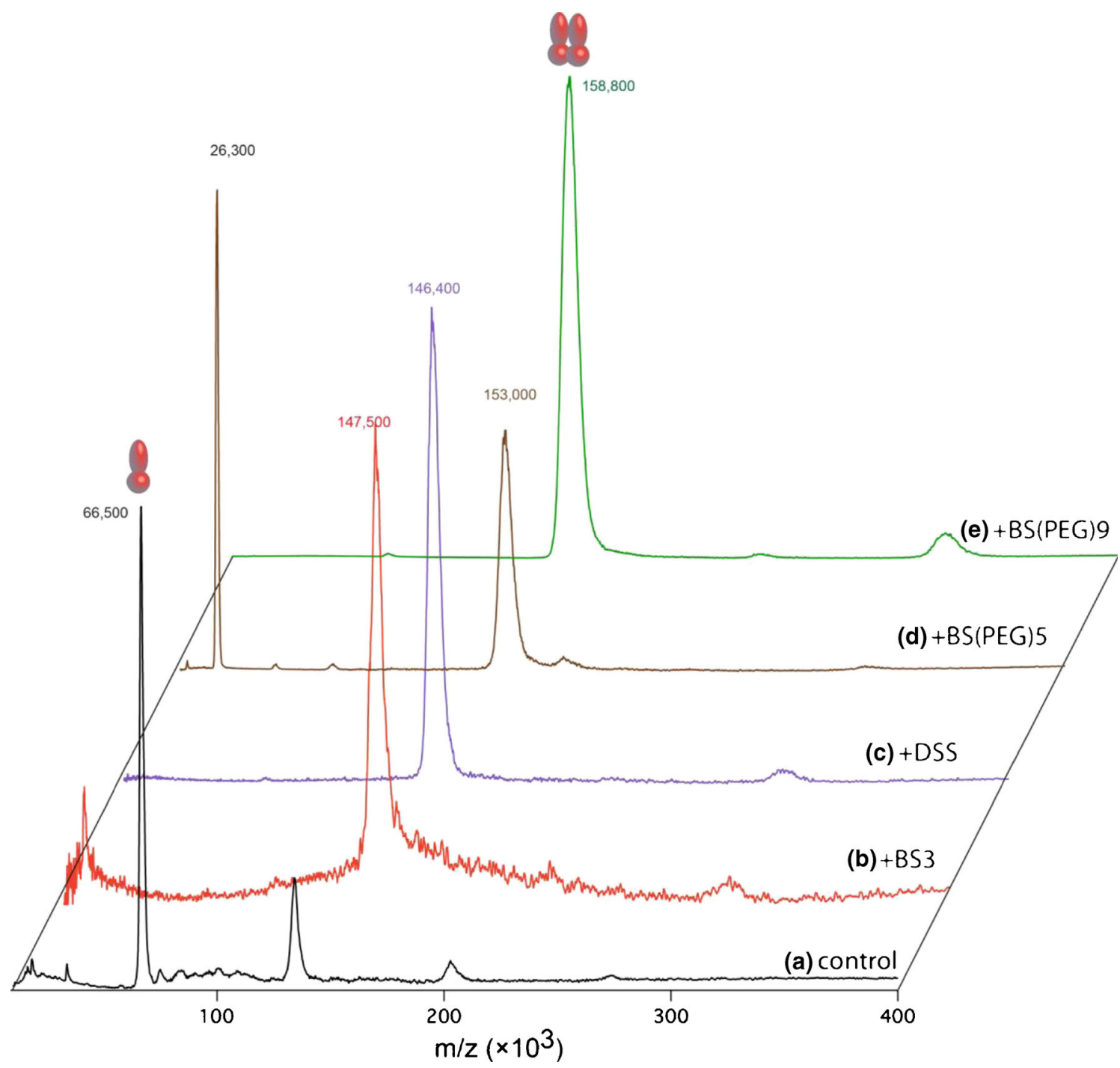

Figure 2. High-mass MALDI mass spectra of PgIK in C12E8 (a) without any cross-linker, and after applying BS ${ }^{3}$ (b), DSS (c), $\mathrm{BS}(\mathrm{PEG})_{5}(\mathbf{d})$, and $\mathrm{BS}(\mathrm{PEG})_{9}(\mathbf{e})$, respectively

$\mathrm{BS}(\mathrm{PEG})_{5}$ (Figure 3d) did we observe peaks attributable to the complex, including signals at $\mathrm{m} / \mathrm{z}$ 67,100 (BtuCD), 95,100 $\left(\mathrm{BtuCD}_{2}\right), 133,700\left(\mathrm{BtuC}_{2} \mathrm{D}_{2}\right)$, along with a higher intensity of the BtuD dimer peak at $m / z 57,000$ and of the BtuC monomer at $m / z 38,600$, as shown in Figure 3d. Similarly, after introducing $\mathrm{BS}(\mathrm{PEG})_{9}$ (Figure $\left.3 \mathrm{e}\right)$, the $\mathrm{BtuC}_{2} \mathrm{D}_{2}$ complex was stabilized, as seen from observing the species at $\mathrm{m} / \mathrm{z} 68,800$ (BtuCD), 97,700 (BtuCD 2$)$, and 136,800 $\left(\mathrm{BtuC}_{2} \mathrm{D}_{2}\right)$.

For PglK, the cross-linking efficiency was comparable for all NHS esters and for glutaraldehyde. Supplementary Table 2 presents the cross-linking efficiency of all NHS esters applied to PglK, which was solubilized in two different detergent micelle preparations. In both detergents, all the cross-linkers exhibited a similar cross-linking efficiency, 63\% in DDM and $71 \%$ in $\mathrm{C} 12 \mathrm{E} 8$. In a previous report, comparable cross-linking efficiency was observed for NCoA-1·STAT6Y, which has a $\mathrm{K}_{\mathrm{D}}$ around $30 \mathrm{nM}$ [21]. This suggests that the $\mathrm{PglK}$ dimer is also tightly bound, with a $\mathrm{K}_{\mathrm{D}}$ in the nanomolar range. Besides the contribution of the binding affinity of the PglK dimer, we also believe the high prevalence of lysine residues in PglK (55 lysine residues, 9.4\%, higher than average, which is around $6 \%$ ), to play an important role in the stabilization via chemical cross-linking.

As opposed to the high cross-linking efficiency of all NHS esters in stabilizing the PglK complex, neither $\mathrm{BS}^{3}$ nor DSS was able to stabilize the $\mathrm{BtuC}_{2} \mathrm{D}_{2}$ complex. $\mathrm{BS}(\mathrm{PEG})_{5}$ or $\mathrm{BS}(\mathrm{PEG})_{9}$ could stabilize the $\mathrm{BtuC}_{2} \mathrm{D}_{2}$ complex, but with cross-linking efficiency much lower than that of glutaraldehyde [4]. We suggest that the difference in efficiency of the different NHS cross-linkers in stabilizing the $\mathrm{BtuC}_{2} \mathrm{D}_{2}$ complex is due to the different spacer arm lengths: DSS has a carbon chain with a length of only $11.4 \AA$, whereas $\mathrm{BS}(\mathrm{PEG})_{5}$ has a $21.7 \AA$ and $\mathrm{BS}(\mathrm{PEG})_{9}$ a $35.8 \AA$ spacer arm length. In our previous work, we have found that the cross-linking efficiency for soluble protein complexes is strongly influenced by the distances of target amino acid residues [22]. Therefore, we calculated the distances between primary amine groups, including the $\mathrm{N}$ terminus and the Lys residues, in the different subunits of 


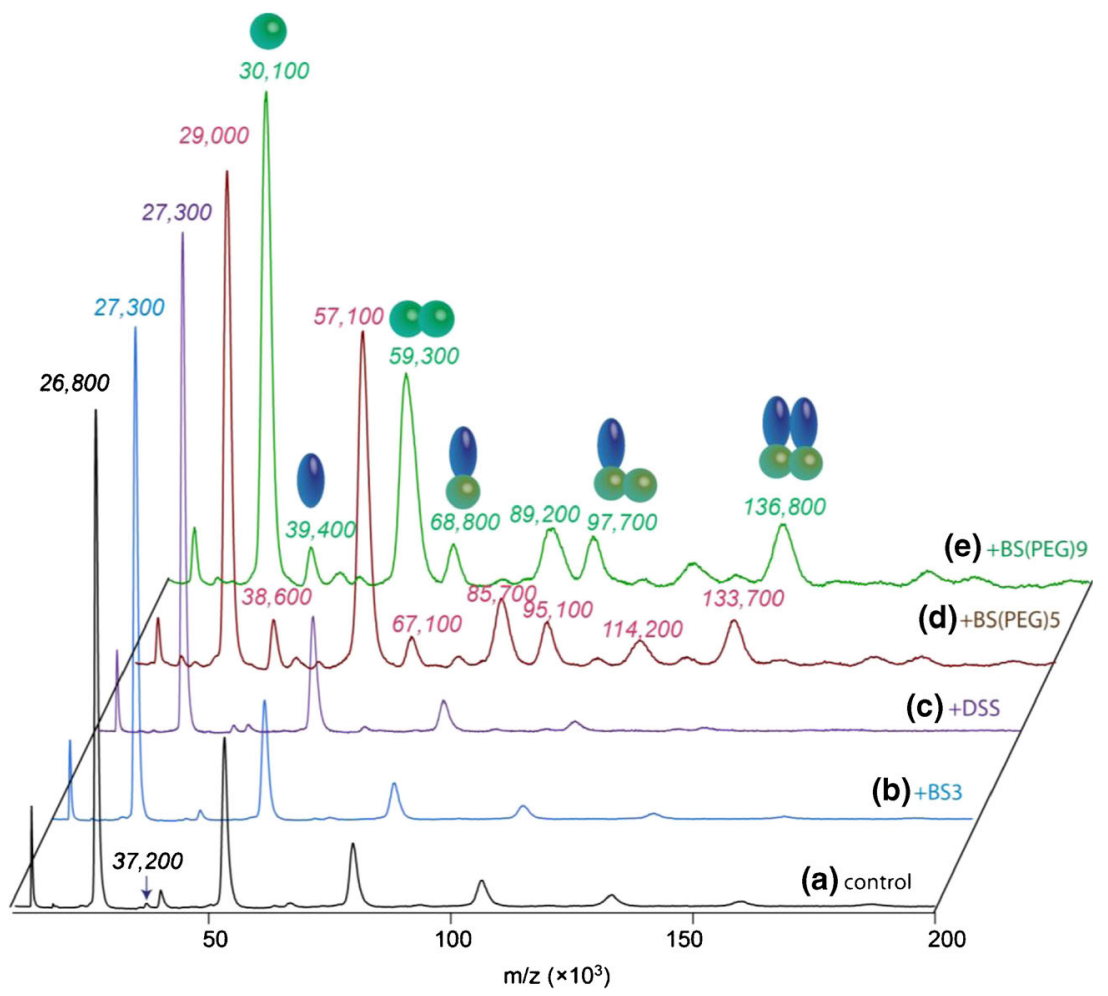

Figure 3. High-mass MALDI mass spectra of $\mathrm{BtuC}_{2} \mathrm{D}_{2}$ in LDAO (a) without any cross-linker (control), and after applying $B S^{3}$ (b), DSS (c), BS(PEG) $)_{5}(\mathbf{d})$, and BS(PEG) $)_{9}(\mathbf{e})$, respectively. Blue symbolizes the BtuC subunit and green the BtuD subunit

BtuC $_{2} \mathrm{D}_{2}$ from its crystal structure (PDB 1L7V, Figure 4a). We plotted the distances between primary amine groups in the $\mathrm{B}$ subunit (BtuC in green) and those in the other three units in Figure $4 \mathrm{~b}$. The B subunit has only two primary amine groups, one lysine residue and the N-terminus. Distances to other amine groups in the complex are mostly in the 25 to $70 \AA$ range (Figure 4b); distances below $20 \AA$ (12.3 and 18.2 $\AA$ ) are only found in two Lys residues in the $\mathrm{D}$ subunit (BtuD). For the $\mathrm{C}$ subunit (BtuD in pink), the same evaluation is presented in Figure 4c. For the target amine groups in the D subunit (BtuD subunit), distances below $20 \AA$ to the amine groups in other subunits are mostly found within the other BtuD subunit. This

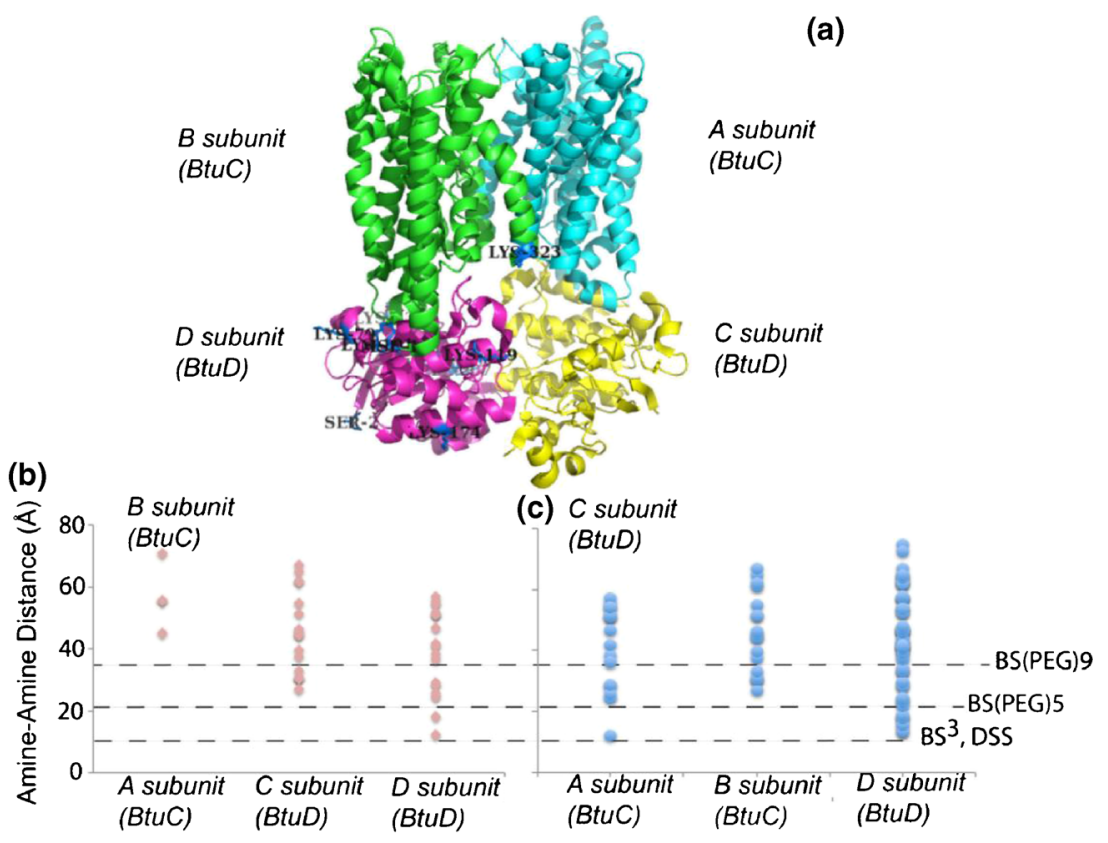

Figure 4. Ribbon diagram of BtuCD (PDB ID code 1L7V) (a); distribution of inter-subunit amine-amine distances between the B

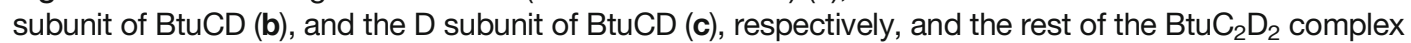


explains the observation of the higher BtuD dimer intensity compared with other subcomplexes, such as $\mathrm{BtuCD}, \mathrm{BtuCD}_{2}$, or the full $\mathrm{BtuC}_{2} \mathrm{D}_{2}$. The amine-amine distances thus dictate whether cross-linkers such as $\mathrm{BS}(\mathrm{PEG})_{5}$ or $\mathrm{BS}(\mathrm{PEG})_{9}$ are able to stabilize the $\mathrm{BtuC}_{2} \mathrm{D}_{2}$ complex or not, as in the case of DSS. Moreover, we hypothesize that the limited number of lysine residues in the BtuC subunit results in a low cross-linking efficiency, even when $\mathrm{BS}(\mathrm{PEG})_{5}$ or $\mathrm{BS}(\mathrm{PEG})_{9}$ are used. If specific cross-linkers of defined length are employed, subsequent digestion of a protein or a protein complex can yield distance constraints on the three-dimensional structure. The correlation between the chemical cross-linking efficiency and the spacer arm length of various NHS ester-based cross-linkers could, in the future, be exploited to map membrane protein structures, even in the presence of detergent micelles.

\section{Conclusions}

In this study, we conducted cross-linking with chemically specific NHS-esters of two membrane protein complexes, PglK and $\mathrm{BtuC}_{2} \mathrm{D}_{2}$. The cross-linking experiment was carried out with a series of chemical cross-linkers with different spacer arm lengths. We found clear evidence that NHS esters can be used to stabilize or cross-link membrane protein complexes even in the presence of different detergents, such as DDM, LDAO, and $\mathrm{C} 12 \mathrm{E} 8$. The reactivity differs among the cross-linkers applied, and depends on the protein structure, including the number of lysine residues and the amine-amine distances between different subunits. The low number of available lysine residues is probably the reason for the relatively low cross-linking efficiency in $\mathrm{BtuC}_{2} \mathrm{D}_{2}$, compared with the efficient stabilization of the PglK dimer. The successful cross-linking of membrane protein complexes in different detergent micelles via NHS esters highlights the possibility to map membrane protein structures by chemical cross-linking in situ, combined with mass spectrometry.

\section{Acknowledgments}

The authors acknowledge financial support from the Swiss National Science Foundation (SNF), grant no. 200020124663 (to R.Z.) and grant no. 31003A-116191 (to K.P.L.), as well as from the National Center for Excellence in Research (NCCR) Structural Biology (to K.P.L.).

\section{References}

1. Marcoux, J., Robinson, C.V.: Twenty years of gas phase structural biology. Structure 21, 1541-1550 (2013)
2. Barrera, N.P., Di Bartolo, N., Booth, P.J., Robinson, C.V.: Micelles protect membrane complexes from solution to vacuum. Science 321, 243-246 (2008)

3. Pramanik, A., Hauf, W., Hoffmann, J., Cernescu, M., Brutschy, B., Braun, V.: Oligomeric structure of ExbB and ExbB-ExbD isolated from Escherichia coli as revealed by LILBID mass spectrometry. Biochemistry 50, 8950-8956 (2011)

4. Chen, F., Gerber, S., Heuser, K., Korkhov, V.M., Lizak, C., Mireku, S., Locher, K.P., Zenobi, R.: High-mass matrix-assisted laser desorption ionization-mass spectrometry of integral membrane proteins and their complexes. Anal. Chem. 85, 3483-3488 (2013)

5. Farmer, T.B., Caprioli, R.M.: Determination of protein-protein interactions by matrix-assisted laser desorption/ionization mass spectrometry. J. Mass Spectrom. 33, 697-704 (1998)

6. Pimenova, T., Pereira, C.P., Schaer, D.J., Zenobi, R.: Characterization of high molecular weight multimeric states of human haptoglobin and hemoglobin-based oxygen carriers by high-mass MALDI MS. J. Sep. Sci. 32, 1224-1230 (2009)

7. Migneault, I., Dartiguenave, C., Bertrand, M.J., Waldron, K.C.: Glutaraldehyde: behavior in aqueous solution, reaction with proteins, and application to enzyme crosslinking. BioTechniques 37, 790-802 (2004)

8. Mädler, S., Bich, C., Touboul, D., Zenobi, R.: Chemical cross-linking with NHS esters: a systematic study on amino acid reactivities. J. Mass Spectrom. 44, 694-706 (2009)

9. Sinz, A.: Chemical cross-linking and mass spectrometry to map threedimensional protein structures and protein-protein interactions. Mass Spectrom. Rev. 25(4), 663-682, (2006)

10. Sinz, A.: Chemical cross-linking and mass spectrometry for mapping threedimensional structures of proteins and protein complexes. J. Mass Spectrom. 38, 1225-1237 (2003)

11. Kalkhof, S., Sinz, A.: Chances and pitfalls of chemical cross-linking with amine-reactive $N$-hydroxysuccinimide esters. Anal. Bioanal. Chem. 392, 305-312 (2008)

12. Müller, M.Q., Sinz, A.: Chemical cross-linking and high-resolution mass spectrometry to study protein-drug interactions. Methods Mol. Biol. 803, 205-218 (2012)

13. Zhang, H., Tang, X., Munske, G.R., Tolic, N., Anderson, G.A., Bruce, J.E.: In vivo identification of the outer membrane protein OmcA-MtrC interaction network in Shewanella oneidensis MR-1 cells using novel hydrophobic chemical cross-linkers. Mol. Cell. Proteomics 8, 409-420 (2009)

14. Zheng, C., Yang, L., Hoopmann, M.R., Eng, J.K., Tang, X., Weisbrod, C.R., Bruce, J.E.: Cross-linking measurements of in vivo protein complex topologies. Mol. Cell. Proteomics 10(10), M110.006841 (2011)

15. Schmidt, C., Zhou, M., Marriott, H., Morgner, N., Politis, A., Robinson, C.V.: Comparative cross-linking and mass spectrometry of an intact F-type ATPase suggest a role for phosphorylation. Nat. Commun. 4, 1985 (2013)

16. Zhou, M., Robinson, C.V.: Flexible membrane proteins: functional dynamics captured by mass spectrometry. Curr. Opin. Struct. Biol. 28, 122-130 (2014)

17. Locher, K.P.: Review. Structure and mechanism of ATP-binding cassette transporters. Philos. Trans. R. Soc. Lond. B Biol. Sci. 364, 239-245 (2009)

18. Locher, K.P.: Structure and mechanism of ABC transporters. Curr. Opin. Struct. Biol. 14, 426-431 (2004)

19. Dawson, R.J.P., Locher, K.P.: Structure of a bacterial multidrug ABC transporter. Nature 443, 180-185 (2006)

20. Locher, K.P., Lee, A.T., Rees, D.C.: The E. coli BtuCD structure: a framework for $\mathrm{ABC}$ transporter architecture and mechanism. Science 296, 1091-1098 (2002)

21. Mädler, S., Seitz, M., Robinson, J., Zenobi, R.: Does chemical crosslinking with NHS esters reflect the chemical equilibrium of protein-protein noncovalent interactions in solution? J. Am. Soc. Mass Spectrom. 21, 1775-1783 (2010)

22. Chen, F., Nielsen, S., Zenobi, R.: Understanding chemical reactivity for homo- and heterobifunctional protein cross-linking agents. J. Mass Spectrom. 48, 807-812 (2013) 